\title{
A Generalization of the Havrda-Charvat and Tsallis Entropy and Its Axiomatic Characterization
}

\author{
Satish Kumar' and Gurdas Ram ${ }^{2}$ \\ ${ }^{1}$ Department of Mathematics, College of Natural Sciences, Arba Minch University, Arab Minch, Ethiopia \\ ${ }^{2}$ Department of Applied Sciences, Maharishi Markandeshwar University, Solan, Himachal Pradesh 173229, India
}

Correspondence should be addressed to Satish Kumar; drsatish74@rediffmail.com

Received 3 September 2013; Revised 20 December 2013; Accepted 20 December 2013; Published 19 February 2014

Academic Editor: Chengjian Zhang

Copyright (C) 2014 S. Kumar and G. Ram. This is an open access article distributed under the Creative Commons Attribution License, which permits unrestricted use, distribution, and reproduction in any medium, provided the original work is properly cited.

In this communication, we characterize a measure of information of types $\alpha, \beta$, and $\gamma$ by taking certain axioms parallel to those considered earlier by Havrda and Charvat along with the recursive relation $H_{n}\left(p_{1}, \ldots, p_{n} ; \alpha, \beta, \gamma\right)-H_{n-1}\left(p_{1}+p_{2}, p_{3}, \ldots, p_{n} ; \alpha, \beta\right.$, $\gamma)=\left(A_{(\alpha, \gamma)} /\left(A_{(\alpha, \gamma)}-A_{(\beta, \gamma)}\right)\right)\left(p_{1}+p_{2}\right)^{\alpha / \gamma} H_{2}\left(p_{1} /\left(p_{1}+p_{2}\right), p_{2} /\left(p_{1}+p_{2}\right) ; \alpha, \gamma\right)+\left(A_{(\beta, \gamma)} /\left(A_{(\beta, \gamma)}-A_{(\alpha, \gamma)}\right)\right)\left(p_{1}+p_{2}\right)^{(\beta / \gamma)} H_{2}\left(p_{1} /\left(p_{1}+p_{2}\right)\right.$, $\left.p_{2} /\left(p_{1}+p_{2}\right) ; \gamma, \beta\right), \alpha \neq \gamma \neq \beta, \alpha, \beta, \gamma>0$. Some properties of this measure are also studied. This measure includes Shannon's information measure as a special case.

\section{Introduction}

Shannon's measure of entropy for a discrete probability distribution

$$
P=\left(p_{1}, \ldots, p_{n}\right), \quad p_{i} \geq 0, \quad \sum_{i=1}^{n} p_{i}=1,
$$

given by

$$
H(P)=-\sum_{i=1}^{n} p_{i} \log p_{i},
$$

has been characterized in several ways (see Aczél and Daróczy [1]). Out of the many ways of characterization the two elegant approaches are to be found in the work of (i) Faddeev [2], who uses branching property namely,

$$
\begin{aligned}
H_{n}\left(p_{1}, \ldots, p_{n}\right)= & H_{n-1}\left(p_{1}+p_{2}, p_{3}, \ldots, p_{n}\right) \\
& +\left(p_{1}+p_{2}\right) H_{2}\left(\frac{p_{1}}{p_{1}+p_{2}}, \frac{p_{2}}{p_{1}+p_{2}}\right),
\end{aligned}
$$

$n=3,4, \ldots$ for the above distribution $P$, as the basic postulate, and (ii) Chaundy and McLeod [3], who studied the functional equation

$$
\sum_{i=1}^{n} \sum_{j=1}^{m} f\left(p_{i} q_{j}\right)=\sum_{i=1}^{n} f\left(p_{i}\right)+\sum_{j=1}^{m} f\left(q_{j}\right),
$$

$$
\text { for } p_{i} \geq 0, q_{j} \geq 0 \text {. }
$$

Both of the above-mentioned approaches have been extensively exploited and generalized. The most general form of (4) has been studied by Sharma and Taneja [4], who considered the functional equation

$$
\begin{aligned}
& \sum_{i=1}^{n} \sum_{j=1}^{m} f\left(p_{i} q_{j}\right)= \sum_{i=1}^{n} \sum_{j=1}^{m} f\left(p_{i}\right) g\left(q_{j}\right) \\
&+\sum_{i=1}^{n} \sum_{j=1}^{m} g\left(p_{i}\right) f\left(q_{j}\right), \\
& \sum_{i=1}^{n} p_{i}=\sum_{j=1}^{m} q_{j}=1, \quad p_{i} \geq 0, q_{j} \geq 0 .
\end{aligned}
$$


We define the information measure as

$$
\begin{aligned}
& H_{n}\left(p_{1}, \ldots, p_{n} ; \alpha, \beta, \gamma\right) \\
& =\left(2^{(\gamma-\alpha) / \gamma}-2^{(\gamma-\beta) / \gamma}\right)^{-1} \sum_{i=1}^{n}\left(p_{i}^{\alpha / \gamma}-p_{i}^{\beta / \gamma}\right), \\
& \alpha \neq \gamma \neq \beta, \alpha, \beta, \gamma>0,
\end{aligned}
$$

for a complete probability distribution $P=\left(p_{1}, \ldots, p_{n}\right), p_{i} \geq$ $0, \sum_{i=1}^{n} p_{i}=1$.

Measure (6) reduces to entropy of type $\beta$ (or $\alpha$ ) when $\alpha=$ $\gamma=1$ (or $\beta=\gamma=1$ ) given by

$$
\begin{array}{r}
H_{n}\left(p_{1}, \ldots, p_{n} ; \beta\right)=\left(2^{1-\beta}-1\right)^{-1}\left[\sum_{i=1}^{n} p_{i}^{\beta}-1\right], \\
\beta \neq 1, \beta>0 .
\end{array}
$$

When $\beta \rightarrow 1$, measure ( 7 ) reduces to Shannon's entropy [5], namely,

$$
H_{n}\left(p_{1}, \ldots, p_{2}\right)=-\sum_{i=1}^{n} p_{i} \log _{2} p_{i} .
$$

The measure (7) was characterized by many authors by different approaches. Havrda and Charvát [6] characterized (7) by an axiomatic approach. Daróczy [7] studied (7) by a functional equation. A joint characterization of the measures (7) and (8) has been done by author in two different ways. Firstly by a generalized functional equation having four different functions and secondly by an axiomatic approach. Later on Tsallis [8] gave the applications of (7) in Physics.

To characterize strongly interacting statistical systems within a thermodynamical framework-complex system in particular-it might be necessary to introduce generalized entropies. A series of such entropies have been proposed in the past, mainly to accommodate important empirical distribution functions to a maximum ignorance principles. The understanding of the fundamental origin of these entropies and its deeper relations to complex systems is limited. Authors [9] explore this question from first principle. Authors [9] observed that the 4th Khinchin axiom is violated by strongly interacting system in general and by assuming the first three Khinchin axioms derived a unique entropy and also classified the known entropies with in equivalence classes.

For statistical system that violates the four ShannonKhinchin axioms, entropy takes a more general form than the Boltzmann-Gibbs entropy. The framework of superstatistics allows one to formulate a maximum entropy principle with these generalized entropies, making them useful for understanding distribution functions of non-Markovian or nonergodic complex systems. For such systems where the composability axiom is violated there exist only two ways to implement the maximum entropy principle; one is using the escort probabilities and the other is not. The two ways are connected through a duality. Authors [10] showed that this duality fixes a unique escort probability and derived a complete theory of the generalized logarithms and also gave the relationship between the functional forms of generalized logarithms and the asymptotic scaling behavior of the entropy.

Suyari [11] has proposed a generalization of ShannonKhinchin axioms, which determines a class of entropies containing the well-known Tsallis and Havrda-Charvat entropies. Authors [12] showed that the class of entropy functions determined by Suyari's axioms is wider than the one proposed by Suyari and generalized Suyari's axioms characterizing recently introduced class of entropies obtained by averaging pseudoadditive information content.

In this communication, we characterized the measure (6) by taking certain axioms parallel to those considered earlier by Havrda and Charvát [6] along with the recursive relation (9). Some properties of this measure are also studied.

The measure (6) satisfies a recursive relation as follows:

$$
\begin{array}{r}
H_{n}\left(p_{1}, \ldots, p_{n} ; \alpha, \beta, \gamma\right)-H_{n-1}\left(p_{1}+p_{2}, p_{3}, \ldots, p_{n} ; \alpha, \beta, \gamma\right) \\
=\frac{A_{(\alpha, \gamma)}}{A_{(\alpha, \gamma)}-A_{(\beta, \gamma)}}\left(p_{1}+p_{2}\right)^{\alpha / \gamma} H_{2}\left(\frac{p_{1}}{p_{1}+p_{2}}, \frac{p_{2}}{p_{1}+p_{2}} ; \alpha, \gamma\right) \\
+\frac{A_{(\beta, \gamma)}}{A_{(\beta, \gamma)}-A_{(\alpha, \gamma)}}\left(p_{1}+p_{2}\right)^{\beta / \gamma} H_{2}\left(\frac{p_{1}}{p_{1}+p_{2}}, \frac{p_{2}}{p_{1}+p_{2}} ; \gamma, \beta\right), \\
\alpha \neq \gamma \neq \beta, \alpha, \beta, \gamma>0,
\end{array}
$$

where $p_{1}+p_{2}>0, \quad A_{(\alpha, \gamma)}=\left(2^{(\gamma-\alpha) / \gamma}-1\right)$, and $A_{(\beta, \gamma)}=$ $\left(2^{(\gamma-\beta) / \gamma}-1\right)$.

Consider

$$
\begin{array}{r}
H\left(p_{1}, p_{2}, \ldots, p_{n} ; \alpha, \gamma\right)=A_{(\alpha, \gamma)}^{-1}\left[\sum_{i=1}^{n} p_{i}^{\alpha / \gamma}-1\right], \\
\alpha \neq \gamma, \alpha, \gamma>0 \neq 1, \\
H\left(p_{1}, p_{2}, \ldots, p_{n} ; \gamma, \beta\right)=A_{(\beta, \gamma)}^{-1}\left[1-\sum_{i=1}^{n} p_{i}^{\beta / \gamma}\right], \\
\beta \neq \gamma, \beta, \gamma>0 \neq 1 .
\end{array}
$$

Proof.

$$
\begin{gathered}
H_{n}\left(p_{1}, \ldots, p_{n} ; \alpha, \beta, \gamma\right)-H_{n-1}\left(p_{1}+p_{2}, p_{3}, \ldots, p_{n} ; \alpha, \beta, \gamma\right) \\
=\left(2^{(\gamma-\alpha) / \gamma}-2^{(\gamma-\beta) / \gamma}\right)^{-1}\left\{\left(p_{1}^{\alpha / \gamma}-p_{1}^{\beta / \gamma}\right)+\left(p_{2}^{\alpha / \gamma}-p_{2}^{\beta / \gamma}\right)\right. \\
\left.+\cdots+\left(p_{n}^{\alpha / \gamma}-p_{n}^{\beta / \gamma}\right)\right\} \\
-\left(2^{(\gamma-\alpha) / \gamma}-2^{(\gamma-\beta) / \gamma}\right)^{-1} \\
\times\left\{\left(p_{1}+p_{2}\right)^{\alpha / \gamma}-\left(p_{1}+p_{2}\right)^{\beta / \gamma}+\left(p_{3}^{\alpha / \gamma}-p_{3}^{\beta / \gamma}\right)\right. \\
\left.+\cdots+\left(p_{n}^{\alpha / \gamma}-p_{n}^{\beta / \gamma}\right)\right\}
\end{gathered}
$$




$$
\begin{aligned}
&=\left(2^{(\gamma-\alpha) / \gamma}-2^{(\gamma-\beta) / \gamma}\right)^{-1}\left\{p_{1}^{\alpha / \gamma}-p_{1}^{\beta / \gamma}+p_{2}^{\alpha / \gamma}-p_{2}^{\beta / \gamma}\right. \\
&\left.-\left(p_{1}+p_{2}\right)^{\alpha / \gamma}+\left(p_{1}+p_{2}\right)^{\beta / \gamma}\right\} \\
&=\left(2^{(\gamma-\alpha) / \gamma}-2^{(\gamma-\beta) / \gamma}\right)^{-1}\left\{p_{1}^{\alpha / \gamma}+p_{2}^{\alpha / \gamma}-\left(p_{1}+p_{2}\right)^{\alpha / \gamma}\right\} \\
&+\left(2^{(\gamma-\alpha) / \gamma}-2^{(\gamma-\beta) / \gamma}\right)^{-1}\left\{\left(p_{1}+p_{2}\right)^{\beta / \gamma}-p_{1}^{\beta / \gamma}-p_{2}^{\beta / \gamma}\right\} \\
&=\left(2^{(\gamma-\alpha) / \gamma}-2^{(\gamma-\beta) / \gamma}\right)^{-1}\left(p_{1}+p_{2}\right)^{\alpha / \gamma} \\
& \times\left[\frac{p_{1}^{\alpha / \gamma}}{\left(p_{1}+p_{2}\right)^{\alpha / \gamma}}+\frac{p_{2}^{\alpha / \gamma}}{\left(p_{1}+p_{2}\right)^{\alpha / \gamma}-1}\right] \\
&+\left(2^{(\gamma-\alpha) / \gamma}-2^{(\gamma-\beta) / \gamma}\right)^{-1}\left(p_{1}+p_{2}\right)^{\beta / \gamma} \\
& \times\left[1-\frac{p_{1}^{\beta / \gamma}}{\left(p_{1}+p_{2}\right)^{\alpha / \gamma}}-\frac{\left(p_{1}+p_{2}\right)^{\alpha / \gamma}}{(\beta / \gamma}\right] \\
&\left.+\frac{A_{(\beta, \gamma)}}{A_{(\beta, \gamma)}-A_{(\alpha, \gamma)}}\left(\frac{p_{1}}{p_{1}+p_{2}}, \frac{p_{2}}{p_{1}+p_{2}} ; \gamma, \beta\right), p_{2}\right)^{\beta / \gamma} \\
& A_{(\alpha, \gamma)}-A_{(\beta, \gamma)}\left(p_{1}+p_{2}\right)^{\alpha / \gamma} H_{2}\left(\frac{p_{1}}{p_{1}+p_{2}}, \frac{p_{2}}{p_{1}+p_{2}} ; \alpha, \gamma\right)
\end{aligned}
$$

which proves (9).

\section{Set of Axioms}

For characterizing a measure of information of types $\alpha, \beta$, and $\gamma$ associated with a probability distribution $P=$ $\left(p_{1}, \ldots, p_{n}\right), p_{i} \geq 0, \sum_{i=1}^{n} p_{i}=1$, we introduce the following axioms:

(1) $H_{n}\left(p_{1}, \ldots, p_{n} ; \alpha, \beta, \gamma\right)$ is continuous in the region

$$
p_{i} \geq 0, \quad \sum_{i=1}^{n} p_{i}=1, \quad \alpha, \beta, \gamma>0
$$

(2) $H_{2}(1,0 ; \alpha, \beta, \gamma)=0$;

(3) $H_{2}(1 / 2,1 / 2 ; \alpha, \beta, \gamma)=1, \alpha, \beta, \gamma>0$;

(4)

$$
\begin{aligned}
& H_{n}\left(p_{1}, \ldots, p_{i-1}, 0, p_{i+1}, \ldots, p_{n} ; \alpha, \beta, \gamma\right) \\
& \quad=H_{n-1}\left(p_{1}, \ldots, p_{i-1}, p_{i+1}, \ldots, p_{n} ; \alpha, \beta, \gamma\right),
\end{aligned}
$$

for every $i=1,2, \ldots, n$;

$$
\begin{array}{r}
H_{n+1}\left(p_{1}, \ldots, p_{i-1}, v_{i_{1}}, v_{i_{2}}, p_{i+1}, \ldots, p_{n} ; \alpha, \beta, \gamma\right) \\
-H_{n}\left(p_{1}, \ldots, p_{i-1}, p_{i}, p_{i+1}, \ldots, p_{n} ; \alpha, \beta, \gamma\right) \\
=\frac{A_{(\alpha, \gamma)}}{A_{(\alpha, \gamma)}-A_{(\beta, \gamma)}} p_{i}^{\alpha / \gamma} H_{2}\left(\frac{v_{i_{1}}}{p_{i}}, \frac{v_{i_{2}}}{p_{i}} ; \alpha, \gamma\right) \\
+\frac{A_{(\beta, \gamma)}}{A_{(\beta, \gamma)}-A_{(\alpha, \gamma)}} p_{i}^{\beta / \gamma} H_{2}\left(\frac{v_{i_{1}}}{p_{i}}, \frac{v_{i_{2}}}{p_{i}} ; \gamma, \beta\right), \\
\alpha \neq \gamma \neq \beta, \alpha, \beta, \gamma>0,
\end{array}
$$

for every $v_{i_{1}}+v_{i_{2}}=p_{i}>0, i=1,2 \ldots, n$, where $A_{(\alpha, \gamma)}=$ $\left(2^{(\gamma-\alpha) / \gamma}-1\right)$ and $A_{(\beta, \gamma)}=\left(2^{(\gamma-\beta) / \gamma}-1\right), \alpha \neq \gamma \neq \beta$.

Theorem 1. If $\alpha \neq \beta \neq \gamma ; \alpha, \beta, \gamma>0$, then the axioms (1)-(5) determine a measure given by

$$
H_{n}\left(p_{1}, \ldots, p_{n} ; \alpha, \beta, \gamma\right)
$$

$$
=\left(A_{(\alpha, \gamma)}-A_{(\beta, \gamma)}\right)^{-1} \sum_{i=1}^{n}\left(p_{i}^{\alpha / \gamma}-p_{i}^{\beta / \gamma}\right),
$$

$$
\alpha \neq \gamma \neq \beta, \alpha, \beta, \gamma>0
$$

where $A_{(\alpha, \gamma)}=\left(2^{(\gamma-\alpha) / \gamma}-1\right)$ and $A_{(\beta, \gamma)}=\left(2^{(\gamma-\beta) / \gamma}-1\right)$.

Before proving the theorem we prove some intermediate results based on the above axioms.

Lemma 2. If $v_{k} \geq 0, k=1,2, \ldots, m$ and $\sum_{k=1}^{m} v_{k}=p_{i}>0$, then

$$
\begin{aligned}
H_{n+m-1} & \left(p_{1}, \ldots, p_{i-1}, v_{1}, \ldots, v_{m}, p_{i+1}, \ldots, p_{n} ; \alpha, \beta, \gamma\right) \\
= & H_{n}\left(p_{1}, \ldots, p_{n} ; \alpha, \beta, \gamma\right) \\
& +\frac{A_{(\alpha, \gamma)}}{A_{(\alpha, \gamma)}-A_{(\beta, \gamma)}} p_{i}^{\alpha / \gamma} H_{m}\left(\frac{v_{1}}{p_{i}}, \ldots, \frac{v_{m}}{p_{i}} ; \alpha, \gamma\right) \\
& +\frac{A_{(\beta, \gamma)}}{A_{(\beta, \gamma)}-A_{(\alpha, \gamma)}} p_{i}^{\beta / \gamma} H_{m}\left(\frac{v_{1}}{p_{i}}, \ldots, \frac{v_{m}}{p_{i}} ; \gamma, \beta\right) .
\end{aligned}
$$

Proof. To prove the lemma, we proceed by induction. For $m=$ 2 , the desired statement holds (cf. axiom (4)). Let us suppose 
that the result is true for numbers less than or equal to $m$, we will prove it for $m+1$. We have

$$
\begin{aligned}
& H_{n+m}\left(p_{1}, \ldots, p_{i-1}, v_{1}, \ldots, v_{m+1}, p_{i+1}, \ldots, p_{n} ; \alpha, \beta, \gamma\right) \\
& =H_{n+1}\left(p_{1}, \ldots, p_{i-1}, v_{1}, L, p_{i+1}, \ldots, p_{n} ; \alpha, \beta, \gamma\right) \\
& +\frac{A_{(\alpha, \gamma)}}{A_{(\alpha, \gamma)}-A_{(\beta, \gamma)}} L^{\alpha / \gamma} H_{m}\left(\frac{v_{2}}{L}, \ldots, \frac{v_{m+1}}{L} ; \alpha, \gamma\right) \\
& +\frac{A_{(\beta, \gamma)}}{A_{(\beta, \gamma)}-A_{(\alpha, \gamma)}} L^{\beta / \gamma} H_{m}\left(\frac{v_{2}}{L}, \ldots, \frac{v_{m+1}}{L} ; \gamma, \beta\right) \\
& \text { (where } \left.L=v_{2}+\cdots+v_{m+1}\right) \\
& =H_{n}\left(p_{1}, \ldots, p_{n} ; \alpha, \beta, \gamma\right) \\
& +\frac{A_{(\alpha, \gamma)}}{A_{(\alpha, \gamma)}-A_{(\beta, \gamma)}} p_{i}^{\alpha / \gamma} H_{2}\left(\frac{\nu_{1}}{p_{i}}, \frac{L}{p_{i}} ; \alpha, \gamma\right) \\
& +\frac{A_{(\beta, \gamma)}}{A_{(\beta, \gamma)}-A_{(\alpha, \gamma)}} p_{i}^{\beta / \gamma} H_{2}\left(\frac{\nu_{1}}{p_{i}}, \frac{L}{p_{i}} ; \gamma, \beta\right) \\
& +\frac{A_{(\alpha, \gamma)}}{A_{(\alpha, \gamma)}-A_{(\beta, \gamma)}} L^{\alpha / \gamma} H_{m}\left(\frac{v_{2}}{L}, \ldots, \frac{v_{m+1}}{L} ; \alpha, \gamma\right) \\
& +\frac{A_{(\beta, \gamma)}}{A_{(\beta, \gamma)}-A_{(\alpha, \gamma)}} L^{\beta / \gamma} H_{m}\left(\frac{v_{2}}{L}, \ldots, \frac{v_{m+1}}{L} ; \gamma, \beta\right) \\
& =H_{n}\left(p_{1}, \ldots, p_{n} ; \alpha, \beta, \gamma\right)+\frac{A_{(\alpha, \gamma)}}{A_{(\alpha, \gamma)}-A_{(\beta, \gamma)}} \\
& \times\left\{p_{i}^{\alpha / \gamma} H_{2}\left(\frac{v_{1}}{p_{i}}, \frac{L}{p_{i}} ; \alpha, \gamma\right)\right. \\
& \left.+L^{\alpha / \gamma} H_{m}\left(\frac{v_{2}}{L}, \ldots, \frac{v_{m+1}}{L} ; \alpha, \gamma\right)\right\} \\
& +\frac{A_{(\beta, \gamma)}}{A_{(\beta, \gamma)}-A_{(\alpha, \gamma)}}\left\{p_{i}^{\beta / \gamma} H_{2}\left(\frac{v_{1}}{p_{i}}, \frac{L}{p_{i}} ; \gamma, \beta\right)\right. \\
& \left.+L^{\beta / \gamma} H_{m}\left(\frac{v_{2}}{L}, \ldots, \frac{v_{m+1}}{L} ; \gamma, \beta\right)\right\}, \\
& \text { where } p_{i}=v_{1}+L>0 \text {. }
\end{aligned}
$$

One more application of induction premise yields

$$
\begin{aligned}
H_{m+1} & \left(\frac{v_{1}}{p_{i}}, \ldots, \frac{v_{m+1}}{p_{i}} ; \alpha, \beta, \gamma\right) \\
= & H_{2}\left(\frac{v_{1}}{p_{i}}, \frac{L}{p_{i}} ; \alpha, \beta, \gamma\right) \\
& +\frac{A_{(\alpha, \gamma)}}{A_{(\alpha, \gamma)}-A_{(\beta, \gamma)}}\left(\frac{L}{p_{i}}\right)^{\alpha / \gamma} H_{m}\left(\frac{v_{2}}{L}, \ldots, \frac{v_{m+1}}{L} ; \alpha, \gamma\right) \\
& +\frac{A_{(\alpha, \gamma)}}{A_{(\alpha, \gamma)}-A_{(\beta, \gamma)}}\left(\frac{L}{p_{i}}\right)^{\beta / \gamma} H_{m}\left(\frac{v_{2}}{L}, \ldots, \frac{v_{m+1}}{L} ; \gamma, \beta\right) .
\end{aligned}
$$

For $\beta=\gamma,(18)$ reduces to

$$
\begin{aligned}
& H_{m+1}\left(\frac{v_{1}}{p_{i}}, \ldots, \frac{v_{m+1}}{p_{i}} ; \alpha, \gamma\right) \\
& \quad=H_{2}\left(\frac{v_{1}}{p_{i}}, \frac{L}{p_{i}} ; \alpha, \gamma\right)+\left(\frac{L}{p_{i}}\right)^{\alpha / \gamma} H_{m}\left(\frac{v_{2}}{L}, \ldots, \frac{v_{m+1}}{L} ; \alpha, \gamma\right)
\end{aligned}
$$

Similarly for $\alpha=\gamma,(18)$ reduces to

$$
\begin{aligned}
& H_{m+1}\left(\frac{v_{1}}{p_{i}}, \ldots, \frac{v_{m+1}}{p_{i}} ; \gamma, \beta\right) \\
& \quad=H_{2}\left(\frac{v_{1}}{p_{i}}, \frac{L}{p_{i}} ; \gamma, \beta\right)+\left(\frac{L}{p_{i}}\right)^{\beta / \gamma} H_{m}\left(\frac{v_{2}}{L}, \ldots, \frac{v_{m+1}}{L} ; \gamma, \beta\right) .
\end{aligned}
$$

Expression (17) together with (19) and (20) gives the desired result.

Lemma 3. If $v_{i j} \geq 0, j=1,2, \ldots, m_{i}, \sum_{j=1}^{m_{i}} v_{i j}=p_{i}>0$, $i=1,2, \ldots, n$, and $\sum_{i=1}^{n} p_{i}=1$, then

$$
\begin{aligned}
& H_{m_{1}+\cdots+m_{n}}\left(v_{11}, v_{12}, \ldots, v_{1 m_{1}}: \cdots: v_{n 1},\right. \\
& \left.={ }_{n 2}, \ldots, v_{n m_{n}} ; \alpha, \beta, \gamma\right) \\
& +\frac{A_{(\alpha, \gamma)}}{A_{(\alpha, \gamma)}-A_{(\beta, \gamma)}} \sum_{i=1}^{n} p_{i}^{\alpha / \gamma} H_{m_{i}}\left(\frac{v_{i 1}}{p_{i}}, \ldots, \frac{v_{i m_{i}}}{p_{i}} ; \alpha, \gamma\right) \\
& +\frac{A_{(\beta, \gamma)}}{A_{(\beta, \gamma)}-A_{(\alpha, \gamma)}} \sum_{i=1}^{n} p_{i}^{\beta / \gamma} H_{m_{i}}\left(\frac{v_{i 1}}{p_{i}}, \ldots, \frac{v_{i m_{i}}}{p_{i}} ; \gamma, \beta\right) .
\end{aligned}
$$

Proof. Proof of this lemma directly follows from Lemma 2.

Lemma 4. If $F(n ; \alpha, \beta, \gamma)=H_{n}(1 / n, \ldots, 1 / n ; \alpha, \beta, \gamma)$, then

$$
\begin{aligned}
F(n ; \alpha, \beta, \gamma)= & \frac{A_{(\alpha, \gamma)}}{A_{(\alpha, \gamma)}-A_{(\beta, \gamma)}} F(n ; \alpha, \gamma) \\
& +\frac{A_{(\beta, \gamma)}}{A_{(\beta, \gamma)}-A_{(\alpha, \gamma)}} F(n ; \gamma, \beta),
\end{aligned}
$$

where $F(n ; \alpha, \gamma)=A_{(\alpha, \gamma)}^{-1}\left(n^{(\gamma-\alpha) / \gamma}-1\right), \alpha \neq \gamma$, and

$$
F(n ; \gamma, \beta)=A_{(\beta, \gamma)}^{-1}\left(n^{(\gamma-\beta) / \gamma}-1\right), \quad \beta \neq \gamma .
$$


Proof. Replacing in Lemma $3 m_{i}$ by $m$ and putting $v_{i j}=$ $1 / m n, i=1,2, n, j=1,2, m$, where $m$ and $n$ are positive integer, we have

$$
\begin{aligned}
F(m n ; \alpha, \beta, \gamma)= & F(m ; \alpha, \beta, \gamma) \\
& +\frac{A_{(\alpha, \gamma)}}{A_{(\alpha, \gamma)}-A_{(\beta, \gamma)}}\left(\frac{1}{m}\right)^{(\alpha-\gamma) / \gamma} F(n ; \alpha, \gamma) \\
& +\frac{A_{(\beta, \gamma)}}{A_{(\beta, \gamma)}-A_{(\alpha, \gamma)}}\left(\frac{1}{m}\right)^{(\beta-\gamma) / \gamma} F(n ; \gamma, \beta),
\end{aligned}
$$

$$
\begin{aligned}
F(m n ; \alpha, \beta, \gamma)= & F(n ; \alpha, \beta, \gamma) \\
& +\frac{A_{(\alpha, \gamma)}}{A_{(\alpha, \gamma)}-A_{(\beta, \gamma)}}\left(\frac{1}{n}\right)^{(\alpha-\gamma) / \gamma} F(m ; \alpha, \gamma) \\
& +\frac{A_{(\beta, \gamma)}}{A_{(\beta, \gamma)}-A_{(\alpha, \gamma)}}\left(\frac{1}{n}\right)^{\beta / \gamma-1} F(m ; \gamma, \beta) .
\end{aligned}
$$

Putting $m=1$ in (24) and using $F(1 ; \alpha, \beta, \gamma)=0$ (by axiom (2)), we get

$$
\begin{aligned}
F(n ; \alpha, \beta, \gamma)= & \frac{A_{(\alpha, \gamma)}}{A_{(\alpha, \lambda)}-A_{(\beta, \gamma)}} F(n ; \alpha, \gamma) \\
& +\frac{A_{(\beta, \gamma)}}{A_{(\beta, \gamma)}-A_{(\alpha, \gamma)}} F(n ; \gamma, \beta),
\end{aligned}
$$

which is (22).

Comparing the right hand sides of (24) and (25), we get

$$
\begin{aligned}
& F(m ; \alpha, \beta, \gamma)+\frac{A_{(\alpha, \gamma)}}{A_{(\alpha, \gamma)}-A_{(\beta, \gamma)}}\left(\frac{1}{m}\right)^{\alpha /(\alpha-\gamma)} F(n ; \alpha, \gamma) \\
& +\frac{A_{(\beta, \gamma)}}{A_{(\beta, \gamma)}-A_{(\alpha, \gamma)}}\left(\frac{1}{m}\right)^{\beta /(\beta-\gamma)} F(n ; \gamma, \beta) \\
& =F(n ; \alpha, \beta, \gamma)+\frac{A_{(\alpha, \gamma)}}{A_{(\alpha, \gamma)}-A_{(\beta, \gamma)}}\left(\frac{1}{n}\right)^{\alpha /(\alpha-\gamma)} F(m ; \alpha, \gamma) \\
& +\frac{A_{(\beta, \gamma)}}{A_{(\beta, \gamma)}-A_{(\alpha, \gamma)}}\left(\frac{1}{n}\right)^{\beta /(\beta-\gamma)} F(m ; \gamma, \beta) .
\end{aligned}
$$

Equation (27) together with (22) gives

$$
\begin{aligned}
A_{(\alpha, \gamma)}\left\{\left[1-\left(\frac{1}{n}\right)^{\alpha / \gamma-1}\right] F(m ; \alpha, \gamma)\right. \\
\left.+\left[\left(\frac{1}{m}\right)^{\alpha / \gamma-1}-1\right] F(n ; \alpha, \gamma)\right\} \\
=A_{(\beta, \gamma)}\left\{\left[1-\left(\frac{1}{n}\right)^{\beta / \gamma-1}\right] F(m ; \gamma, \beta)\right. \\
\left.+\left[\left(\frac{1}{m}\right)^{\beta / \gamma-1}-1\right] F(n ; \gamma, \beta)\right\} .
\end{aligned}
$$

Putting $n=2$ in (28) and using $F(2, \alpha, \beta, \gamma)=$ $H_{2}(1 / 2,1 / 2 ; \alpha, \beta, \gamma)=1$, we get

$$
\begin{aligned}
& A_{(\alpha, \gamma)}\left\{\left(1-2^{1-\alpha / \gamma}\right) F(m ; \alpha, \gamma)-\left(1-\left(\frac{1}{m}\right)^{\alpha / \gamma-1}\right)\right\} \\
& =A_{(\beta, \gamma)}\left\{\left(1-2^{1-\beta / \gamma}\right) F(m ; \gamma, \beta)-\left(1-\left(\frac{1}{m}\right)^{\beta / \gamma-1}\right)\right\} \\
& =C \text { (say). }
\end{aligned}
$$

That is, $A_{(\alpha, \gamma)}\left\{\left(1-2^{1-\alpha / \gamma}\right) F(m ; \alpha, \gamma)-\left(1-(1 / m)^{\alpha / \gamma-1}\right)\right\}=C$, where $C$ is an arbitrary constant.

For $m=1$, we get $C=0$.

Thus, we have

$$
F(m ; \alpha, \gamma)=\frac{1-m^{1-\alpha / \gamma}}{1-2^{1-\alpha / \gamma}}=A_{(\alpha, \gamma)}^{-1}\left(m^{1-\alpha / \gamma}-1\right), \quad \alpha \neq \gamma .
$$

Similarly,

$$
F(m ; \gamma, \beta)=\frac{1-m^{1-\beta / \gamma}}{1-2^{1-\beta / \gamma}}=A_{(\beta, \gamma)}^{-1}\left(m^{1-\beta / \gamma}-1\right), \quad \beta \neq \gamma,
$$

which is (23).

Now (22) together with (23) gives

$$
\begin{aligned}
F(n ; \alpha, \beta, \gamma)= & \frac{A_{(\alpha, \gamma)}}{A_{(\alpha, \gamma)}-A_{(\beta, \gamma)}} F(n ; \alpha, \gamma) \\
& +\frac{A_{(\beta, \gamma)}}{A_{(\beta, \gamma)}-A_{(\alpha, \gamma)}} F(n ; \gamma, \beta) \\
= & \left(A_{(\alpha, \gamma)}-A_{(\beta, \gamma)}\right)^{-1}\left(n^{1-\alpha / \gamma}-n^{1-\beta / \gamma}\right) .
\end{aligned}
$$

Proof of the Theorem. We prove the theorem for rationals and then the continuity axiom (1) extends the result for reals. For this let $m$ and $r_{i}^{\prime}$ s be positive integers such that $\sum_{i=1}^{n} r_{i}=m$ and if we put $p_{i}=r_{i} / m, i=1,2, \ldots, n$ then an application of Lemma 3 gives

$$
\begin{aligned}
& H_{m}(\underbrace{\frac{1}{m}, \ldots, \frac{1}{m}}_{r_{1}}, \ldots, \underbrace{\frac{1}{m}, \ldots, \frac{1}{m}}_{r_{n}} ; \alpha, \beta, \gamma) \\
& =H_{n}\left(p_{1}, p_{2}, \ldots, p_{n} ; \alpha, \beta, \gamma\right) \\
& +\frac{A_{(\alpha, \gamma)}}{A_{(\alpha, \gamma)}-A_{(\beta, \gamma)}} \sum_{i=1}^{n} p_{i}^{\alpha / \gamma} H_{r_{i}}\left(\frac{1}{r_{i}}, \ldots, \frac{1}{r_{i}} ; \alpha, \gamma\right) \\
& +\frac{A_{(\beta, \gamma)}}{A_{(\beta, \gamma)}-A_{(\alpha, \gamma)}} \sum_{i=1}^{n} p_{i}^{\beta / \gamma} H_{r_{i}}\left(\frac{1}{r_{i}}, \ldots, \frac{1}{r_{i}} ; \gamma, \beta\right) .
\end{aligned}
$$


That is,

$$
\begin{aligned}
H_{n} & \left(p_{1}, \ldots, p_{n} ; \alpha, \beta, \gamma\right) \\
= & F(m ; \alpha, \beta, \gamma) \\
& -\frac{A_{(\alpha, \gamma)}}{A_{(\alpha, \gamma)}-A_{(\beta, \gamma)}} \sum_{i=1}^{n} p_{i}^{\alpha / \gamma} F\left(r_{i} ; \alpha, \gamma\right) \\
& -\frac{A_{(\beta, \gamma)}}{A_{(\beta, \gamma)}-A_{(\alpha, \gamma)}} \sum_{i=1}^{n} p_{i}^{\beta / \gamma} F\left(r_{i} ; \gamma, \beta\right) .
\end{aligned}
$$

Equation (34) together with (23) and (32) gives

$$
\begin{array}{r}
H_{n}\left(p_{1}, \ldots, p_{n} ; \alpha, \beta, \gamma\right)=\frac{1}{A_{(\alpha, \gamma)}-A_{(\beta, \gamma)}} \sum_{i=1}^{n}\left(p_{i}^{\alpha / \gamma}-p_{i}^{\beta / \gamma}\right), \\
\alpha \neq \gamma \neq \beta, \alpha, \beta, \gamma>0 .
\end{array}
$$

which is (15).

This completes the proof of the theorem.

\section{Properties of Entropy of Types $\alpha$, $\beta$, and $\gamma$}

The measure $H_{n}(P ; \alpha, \beta, \gamma)$, where $P=\left(p_{1}, \ldots, p_{n}\right), p_{i} \geq 0$, $\sum_{i=1}^{n} p_{i}=1$, is a probability distribution, as characterized in the preceding section and satisfies certain properties, which are given in the following theorems:

Theorem 5. The measure $H_{n}(P ; \alpha, \beta, \gamma)$ is nonnegative for $\alpha \neq \gamma \neq \beta, \alpha, \beta, \gamma>0$.

Proof.

Case 1. $\alpha>\gamma ; \beta<\gamma \Rightarrow \alpha / \gamma>1, \beta / \lambda<1$;

$$
\begin{aligned}
& \Longrightarrow \sum_{i=1}^{n} p_{i}^{\alpha / \gamma}<1, \quad \sum_{i=1}^{n} p_{i}^{\beta / \gamma}>1, \\
& \Longrightarrow \sum_{i=1}^{n}\left(p_{i}^{\alpha / \gamma}-p_{i}^{\beta / \gamma}\right)<0 .
\end{aligned}
$$

Since, $\alpha>\gamma$ and $\beta<\gamma$, we get

$$
\left(2^{1-\alpha / \gamma}-2^{1-\beta / \gamma}\right)^{-1} \sum_{i=1}^{n}\left(p_{i}^{\alpha / \gamma}-p_{i}^{\beta / \gamma}\right)>0 .
$$

Case 2. Similarly for $\alpha<\gamma$ and $\beta>\gamma$, we get

$$
\left(2^{1-\alpha / \gamma}-2^{1-\beta / \gamma}\right)^{-1} \sum_{i=1}^{n} p_{i}^{\alpha / \gamma}-p_{i}^{\beta / \gamma}>0 .
$$

Therefore from Case 1, Case 2, and axiom (2), we get

$$
H_{n}(P ; \alpha, \beta, \gamma) \geq 0
$$

This completes the proof of theorem.
Definition 6. We will use the following definition of a convex function.

A function $f(\cdot)$ over the points in a convex set $R$ is convex $\cap$ if for all $r_{1}, r_{2} \in R$ and $\mu \in(0,1)$

$$
\mu f\left(r_{1}\right)+(1-\mu) f\left(r_{2}\right) \leq f\left(\mu r_{1}+(1-\mu) r_{2}\right) .
$$

The function $f(\cdot)$ is convex $\cup$ if $(40)$ holds with $\geq$ in place of $\leq$

Theorem 7. The measure $H_{n}(P ; \alpha, \beta, \gamma)$ is convex $\cap$ function of the probability distribution $P=\left(p_{1}, \ldots, p_{n}\right), p_{i} \geq 0, \sum_{i=1}^{n} p_{i}=$ 1 , when either $\alpha>$ yand $\beta \leq \gamma$ or $\beta>$ yand $\alpha \leq \gamma$.

Proof. Let there be $r$ distributions

$$
\begin{aligned}
P_{k}(X)=\left\{p_{k}\left(x_{1}\right), \ldots, p_{k}\left(x_{n}\right)\right\}, \quad & \sum_{i=1}^{n} p_{k}\left(x_{i}\right)=1, \\
& k=1,2, \ldots, r
\end{aligned}
$$

associated with the random variable $X=\left(x_{1}, \ldots, x_{n}\right)$.

Consider $r$ numbers $\left(a_{1}, \ldots, a_{r}\right)$ such that $a_{k} \geq 0$ and $\sum_{k=1}^{r} a_{k}=1$ and define

$$
P_{o}(X)=\left\{p_{o}\left(x_{1}\right), \ldots, p_{o}\left(x_{n}\right)\right\},
$$

where

$$
p_{o}\left(x_{i}\right)=\sum_{k=1}^{r} a_{k} p_{k}\left(x_{i}\right), \quad i=1,2, \ldots, n .
$$

Obviously, $\sum_{i=1}^{n} p_{o}\left(x_{i}\right)=1$ and thus $P_{o}(x)$ is a bonafide distribution of $X$.

Let $\alpha>\gamma$ and $0<\beta \leq \gamma$, then we have

$$
\begin{aligned}
& \sum_{k=1}^{r} a_{k} H_{n}\left(p_{k} ; \alpha, \beta, \gamma\right)-H_{n}\left(P_{o}(\alpha, \beta, \gamma)\right) \\
& =\sum_{k=1}^{r} a_{k} H_{n}\left(p_{k} ; \alpha, \beta, \gamma\right) \\
& \quad-\left(A_{(\alpha, \gamma)}-A_{(\beta, \gamma)}\right)^{-1}\left\{\left[\sum_{j=1}^{r} a_{j} p_{j}\right]^{\alpha / \gamma}-\left[\sum_{j=1}^{r} a_{j} p_{j}\right]^{\beta / \gamma}\right\} \\
& \left.\leq \sum_{k=1}^{r} a_{k} H_{n}\left(p_{k} ; \alpha, \beta, \gamma\right)\right\} \\
& \quad-\left(A_{(\alpha, \gamma)}-A_{(\beta, \gamma)}\right)^{-1}\left(\sum_{j=1}^{r} a_{j} p_{j}^{\alpha / \gamma}-\sum_{j=1}^{r} a_{j} p_{j}^{\beta / \gamma}\right)=0,
\end{aligned}
$$

(by Jensen's inequality).

$$
\Rightarrow \sum_{k=1}^{r} a_{k} H_{n}\left(p_{k} ; \alpha, \beta, \gamma\right)-H_{n}\left(P_{o} ; \alpha, \beta, \gamma\right) \leq 0,
$$

that is, $\sum_{k=1}^{r} a_{k} H_{n}\left(p_{k} ; \alpha, \beta, \gamma\right) \leq H_{n}\left(P_{o} ; \alpha, \beta, \gamma\right)$, for $\alpha>\gamma, 0<$ $\beta \leq \gamma$.

By symmetry in $\alpha, \beta$, and $\gamma$ the above result is true for $\beta>\gamma$ and $0<\alpha \leq \gamma$. 
Theorem 8. The measure $H_{n}(p ; \alpha, \beta, \gamma)$ satisfies the following relations:

(i) Generalized-Additive:

$$
\begin{aligned}
& H_{n m}(P * Q ; \alpha, \beta, \gamma)= G_{n}(P ; \alpha, \beta, \gamma) H_{m}(Q ; \alpha, \beta, \gamma) \\
&+G_{m}(Q ; \alpha, \beta, \gamma) H_{n}(P ; \alpha, \beta, \gamma), \\
& \alpha, \beta, \gamma>0,
\end{aligned}
$$

where

$$
\begin{array}{r}
G_{n}(P ; \alpha, \beta, \gamma)=\frac{1}{2} \sum_{i=1}^{n}\left(p_{i}^{\alpha / \gamma}+p_{i}^{\beta / \gamma}\right), \\
\alpha, \beta, \gamma>0 .
\end{array}
$$

(ii) Subadditive: for $\alpha, \beta>\gamma$, the measure $H_{n}(p ; \alpha, \beta, \gamma)$ is subadditive; that is,

$$
\begin{aligned}
H_{n m}(P * Q ; \alpha, \beta, \gamma) \leq & H_{n}(P ; \alpha, \beta, \gamma) \\
& +H_{m}(Q ; \alpha, \beta, \gamma),
\end{aligned}
$$

where $P=\left(p_{1}, \ldots, p_{n}\right), Q=\left(q_{1}, \ldots, q_{m}\right)$ and

$$
P * Q=\left(p_{1} q_{1}, \ldots, p_{1} q_{m}, \ldots, p_{n} q_{1}, \ldots, p_{n} q_{m}\right)
$$

are complete probability distributions.

Proof of (i). We have

$$
\begin{aligned}
& H_{n m}(P * Q ; \alpha, \beta, \gamma)=\left(A_{(\alpha, \gamma)}-A_{(\beta, \gamma)}\right)^{-1} \\
& \times \sum_{i=1}^{n} \sum_{j=1}^{m}\left[\left(p_{i} q_{j}\right)^{\alpha / \gamma}-\left(p_{i} q_{j}\right)^{\beta / \gamma}\right] \\
& =\left(A_{(\alpha, \gamma)}-A_{(\beta, \gamma)}\right)^{-1} \sum_{i=1}^{n} \sum_{j=1}^{m}\left[\left(p_{i} q_{j}\right)^{\alpha / \gamma}-\left(p_{i} q_{j}\right)^{\beta / \gamma}\right. \\
& \left.+p_{i}^{\alpha / \gamma} q_{j}^{\beta / \gamma}-p_{i}^{\alpha / \gamma} q_{j}^{\beta / \gamma}\right] \\
& =\left(A_{(\alpha, \gamma)}-A_{(\beta, \gamma)}\right)^{-1} \sum_{i=1}^{n} \sum_{j=1}^{m}\left[p_{i}^{\alpha / \gamma} q_{j}^{\alpha / \gamma}-p_{i}^{\beta / \gamma} q_{j}^{\beta / \gamma}\right. \\
& \left.\quad+p_{i}^{\alpha / \lambda} q_{j}^{\beta / \gamma}-p_{i}^{\alpha / \gamma} q_{j}^{\beta / \gamma}\right]
\end{aligned}
$$$$
=\left(A_{(\alpha, \gamma)}-A_{(\beta, \gamma)}\right)^{-1} \sum_{i=1}^{n} \sum_{j=1}^{m}\left[p_{i}^{\alpha / \gamma}\left(q_{j}^{\alpha / \gamma}+q_{j}^{\beta / \gamma}\right)\right.
$$$$
\left.-q_{j}^{\beta / \gamma}\left(p_{i}^{\alpha / \gamma}+p_{i}^{\beta / \gamma}\right)\right]
$$

$$
\begin{aligned}
=\left(A_{(\alpha, \gamma)}-A_{(\beta, \gamma)}\right)^{-1} & {\left[\sum_{i=1}^{n} p_{i}^{\alpha / \gamma} \sum_{j=1}^{m}\left(q_{j}^{\alpha / \gamma}+q_{j}^{\beta / \gamma}\right)\right.} \\
& \left.-\sum_{j=1}^{m} q_{j}^{\beta / \gamma} \sum_{i=1}^{n}\left(p_{i}^{\alpha / \gamma}+p_{i}^{\beta / \gamma}\right)\right] .
\end{aligned}
$$

Also

$$
\begin{aligned}
& H_{n m}(P * Q ; \alpha, \beta, \gamma) \\
& =\left(A_{(\alpha, \gamma)}-A_{(\beta, \gamma)}\right)^{-1} \sum_{i=1}^{n} \sum_{j=1}^{m}\left[\left(p_{i} q_{j}\right)^{\alpha / \gamma}-\left(p_{i} q_{j}\right)^{\beta / \gamma}\right] \\
& =\left(A_{(\alpha, \gamma)}-A_{(\beta, \gamma)}\right)^{-1} \sum_{i=1}^{n} \sum_{j=1}^{m}\left[\left(p_{i} q_{j}\right)^{\alpha / \gamma}-\left(p_{i} q_{j}\right)^{\beta / \gamma}\right. \\
& \left.+p_{i}^{\beta / \gamma} q_{j}^{\alpha / \gamma}-p_{i}^{\beta / \gamma} q_{j}^{\alpha / \gamma}\right] \\
& =\left(A_{(\alpha, \gamma)}-A_{(\beta, \gamma)}\right)^{-1} \sum_{i=1}^{n} \sum_{j=1}^{m}\left[p_{i}^{\alpha / \gamma} q_{j}^{\alpha / \gamma}-p_{i}^{\beta / \gamma} q_{j}^{\beta / \gamma}\right. \\
& \left.+p_{i}^{\beta / \gamma} q_{j}^{\alpha / \gamma}-q_{j}^{\alpha / \gamma}\right] \\
& =\left(A_{(\alpha, \gamma)}-A_{(\beta, \gamma)}\right)^{-1} \sum_{i=1}^{n} \sum_{j=1}^{m}\left[q_{j}^{\alpha / \gamma}\left(p_{i}^{\alpha / \gamma}+p_{i}^{\beta / \gamma}\right)\right. \\
& \left.-p_{i}^{\beta / \gamma}\left(q_{j}^{\alpha / \gamma}+q_{j}^{\beta / \gamma}\right)\right] \\
& =\left(A_{(\alpha, \gamma)}-A_{(\beta, \gamma)}\right)^{-1}\left[\sum_{j=1}^{m} q_{j}^{\alpha / \gamma} \sum_{i=1}^{n}\left(p_{i}^{\alpha / \gamma}+p_{i}^{\beta / \gamma}\right)\right. \\
& \left.-\sum_{i=1}^{n} p_{i}^{\beta / \gamma} \sum_{i=1}^{n}\left(q_{j}^{\alpha / \gamma}+q_{j}^{\beta / \gamma}\right)\right] .
\end{aligned}
$$

Adding (49) and (50), we get

$$
\begin{aligned}
2 H_{n m}(P * Q ; \alpha, \beta, \gamma) & \\
=\left(A_{(\alpha, \gamma)}-A_{(\beta, \gamma)}\right)^{-1} & {\left[\sum_{i=1}^{n} p_{i}^{\alpha / \gamma} \sum_{j=1}^{m}\left(q_{j}^{\alpha / \gamma}+q_{j}^{\beta / \gamma}\right)\right.} \\
& \left.-\sum_{j=1}^{m} q_{j}^{\beta / \gamma} \sum_{i=1}^{n}\left(p_{i}^{\alpha / \gamma}+p_{i}^{\beta / \gamma}\right)\right]
\end{aligned}
$$




$$
\begin{aligned}
& +\left(A_{(\alpha, \gamma)}-A_{(\beta, \gamma)}\right)^{-1}\left[\sum_{j=1}^{m} q_{j}^{\alpha / \gamma} \sum_{i=1}^{n}\left(p_{i}^{\alpha / \gamma}+p_{i}^{\beta / \gamma}\right)\right. \\
& \left.\quad-\sum_{i=1}^{n} p_{i}^{\beta / \gamma} \sum_{i=1}^{n}\left(q_{j}^{\alpha / \gamma}+q_{j}^{\beta / \gamma}\right)\right] \\
& =\sum_{i=1}^{n}\left(p_{i}^{\alpha / \gamma}+p_{i}^{\beta / \gamma}\right)\left(A_{(\alpha, \gamma)}-A_{(\beta, \gamma)}\right)^{-1} \\
& \quad \times \sum_{j=1}^{m}\left(q_{j}^{\alpha / \gamma}-q_{j}^{\beta / \gamma}\right) \\
& +\sum_{j=1}^{m}\left(q_{j}^{\alpha / \gamma}+q_{j}^{\beta / \gamma}\right)\left(A_{(\alpha, \gamma)}-A_{(\beta, \gamma)}\right)^{-1} \\
& \quad \times \sum_{i=1}^{n}\left(p_{i}^{\alpha / \gamma}-p_{i}^{\beta / \gamma}\right), \\
& \quad+\sum_{i=1}^{n}\left(p_{i}^{\alpha / \gamma}-p_{i}^{\beta / \gamma}\right) . \\
& \quad+\sum_{j=1}^{m}\left(\sum_{j}^{\alpha / \gamma} * q_{j}^{\beta / \gamma}\right) \\
& +\sum_{i=1}^{n}\left(p_{i}^{\alpha / \gamma}+p_{i}^{\beta / \gamma}\right)\left(A_{(\alpha, \gamma)}-A_{(\beta, \gamma)}\right)^{-1}
\end{aligned}
$$

Using (46)

$$
\begin{aligned}
H_{n m}(P * Q ; \alpha, \beta, s)= & G_{n}(P ; \alpha, \beta, \gamma) H_{m}(Q ; \alpha, \beta, \gamma) \\
& +G_{m}(Q ; \alpha, \beta, \gamma) H_{n}(P ; \alpha, \beta, \gamma),
\end{aligned}
$$

which is (45). This completes the proof of part (i).

Proof of (ii). From part (i), we have

$$
\begin{aligned}
H_{n m}(P * Q ; \alpha, \beta, \gamma)= & G_{n}(P ; \alpha, \beta, \gamma) H_{m}(Q ; \alpha, \beta, \gamma) \\
& +G_{m}(Q ; \alpha, \beta, \gamma) H_{n}(P ; \alpha, \beta, \gamma)
\end{aligned}
$$

$$
\begin{gathered}
\text { As } G_{n}(P ; \alpha, \beta, \gamma)=(1 / 2) \sum_{i=1}^{n}\left(p_{i}^{\alpha / \gamma}+p_{i}^{\beta / \gamma}\right) \leq 1, \text { for } \alpha, \beta \geq \gamma, \\
H_{n m}(P * Q ; \alpha, \beta, \gamma) \leq H_{m}(Q ; \alpha, \beta, \gamma)+H_{n}(P ; \alpha, \beta, \gamma) .
\end{gathered}
$$

\section{Conclusion}

In addition to well-known information measure of Shannon, Renyi's, Havrda-Charvat, Vajda [13], Darcózy, we have characterized a measure which we call $\alpha, \beta$, and $\gamma$ information measure. We have given some basic axioms and properties with recursive relation. The Shannon's [5] measure included in the $\alpha, \beta$, and $\gamma$ information measure for the limiting case $\alpha=\gamma=1$ and $\beta \rightarrow 1 ; \beta=\gamma=1$ and $\alpha \rightarrow 1$. This measure is generalization of Havrda-Charvat entropy.

\section{Conflict of Interests}

The authors declare that there is no conflict of interests regarding the publication of this paper.

\section{References}

[1] J. Aczél and Z. Daróczy, On Measures of Information and Their Characterization, Academic Press, New York, NY, USA, 1975.

[2] D. K. Faddeev, "On the concept of entropy of a finite probabilistic scheme," Uspekhi Matematicheskikh Nauk, vol. 11, no. 1(67), pp. 227-231, 1956.

[3] T. W. Chaundy and J. B. McLeod, "On a functional equation," Proceedings of the Edinburgh Mathematical Society. Series II, vol. 12, no. 43, pp. 6-7, 1960.

[4] B. D. Sharma and I. J. Taneja, "Functional measures in information theory," Funkcialaj Ekvacioj, vol. 17, pp. 181-191, 1974.

[5] C. E. Shannon, "A mathematical theory of communication," The Bell System Technical Journal, vol. 27, pp. 379-423, 623-636, 1948.

[6] J. Havrda and F. Charvát, "Quantification method of classification processes. Concept of structural $\alpha$-entropy," Kybernetika, vol. 3, pp. 30-35, 1967.

[7] Z. Daróczy, “Generalized information functions," Information and Computation, vol. 16, pp. 36-51, 1970.

[8] C. Tsallis, "Possible generalization of Boltzmann-Gibbs statistics," Journal of Statistical Physics, vol. 52, no. 1-2, pp. 479-487, 1988.

[9] R. Hanel and S. Thurner, "A comprehensive classification of complex statistical systems and an ab-initio derivation of their entropy and distribution functions," Europhysics Letters, vol. 93, no. 2, Article ID 20006, 2011.

[10] R. Hanel, S. Thurner, and M. Gell-Mann, "Generalized entropies and logarithms and their duality relations," Proceedings of the National Academy of Sciences of the United States of America, vol. 109, no. 47, pp. 19151-19154, 2012.

[11] H. Suyari, "Generalization of Shannon-Khinchin axioms to nonextensive systems and the uniqueness theorem for the nonextensive entropy," IEEE Transactions on Information Theory, vol. 50, no. 8, pp. 1783-1787, 2004.

[12] V. M. IIic, M. S. Stankovic, and E. H. Mulalic, "Comments on Generalization of Shannon-Khinchin axioms to nonextensive systems and the uniqueness theorem for nonextensive entropy," IEEE Transactions on Information Theory, vol. 59, no. 10, pp. 6950-6952, 2013.

[13] I. Vajda, "Axioms for $\alpha$-entropy of a generalized probability scheme," Kybernetika, vol. 2, pp. 105-112, 1968.

This proves the subadditivity. 


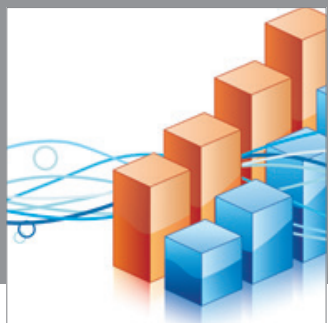

Advances in

Operations Research

mansans

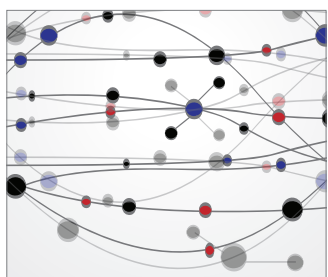

The Scientific World Journal
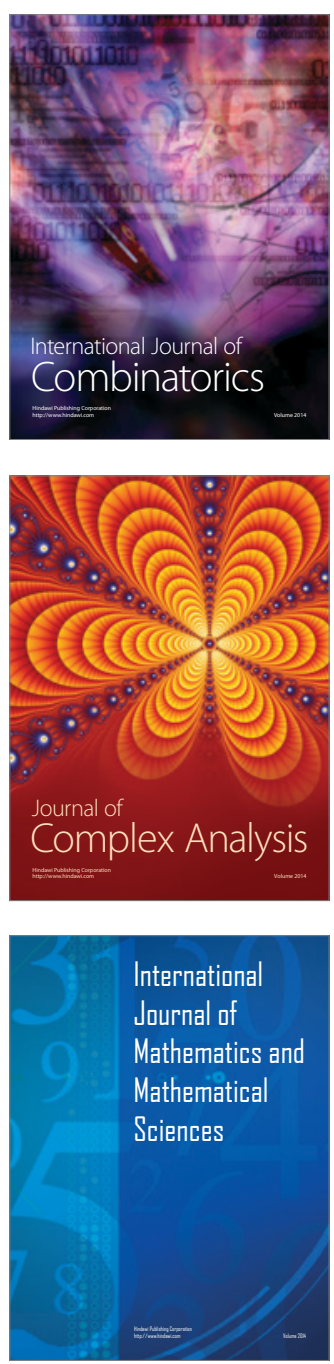
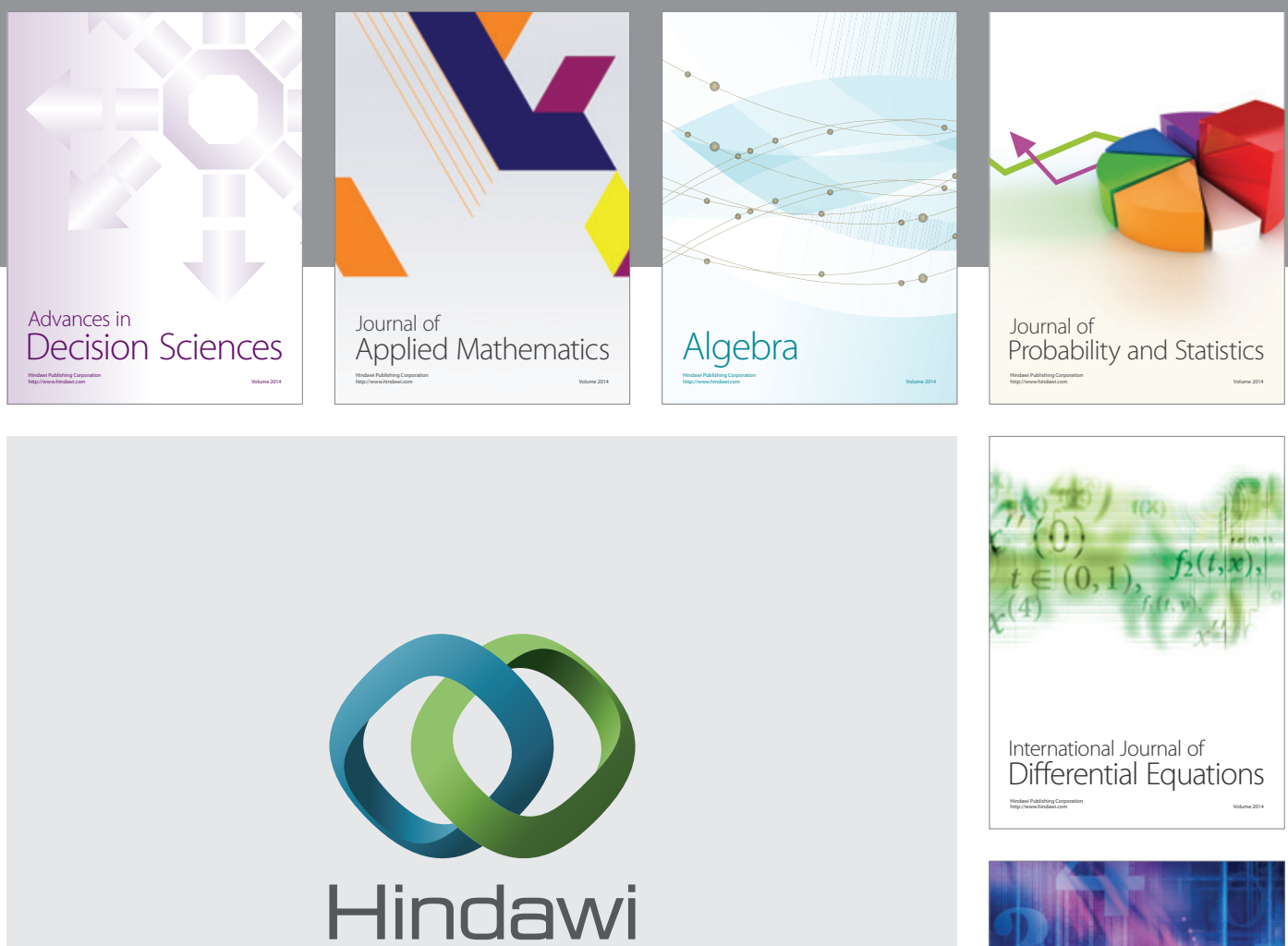

Submit your manuscripts at http://www.hindawi.com
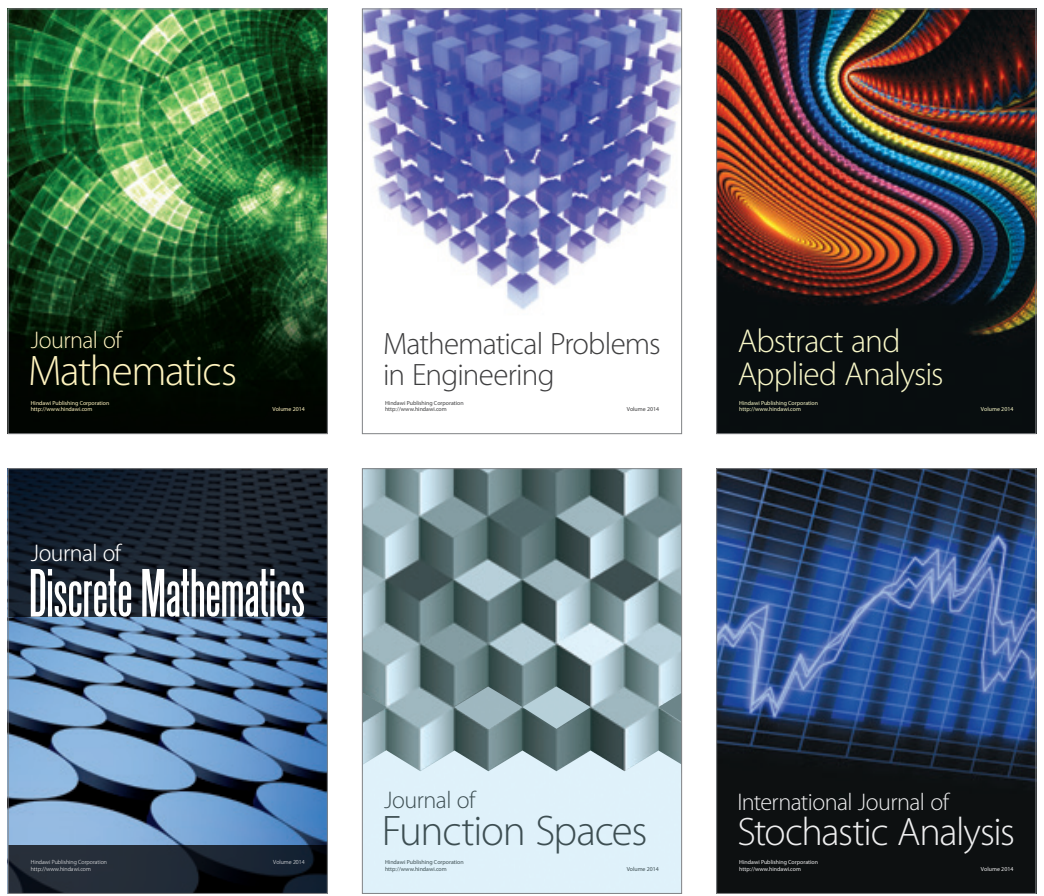

Journal of

Function Spaces

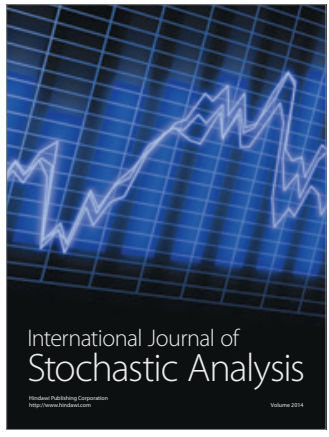

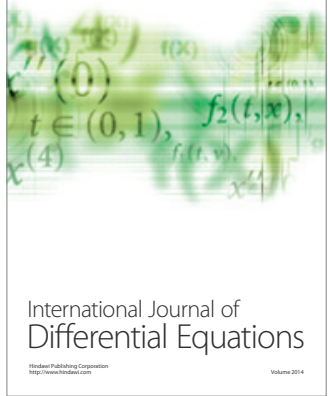
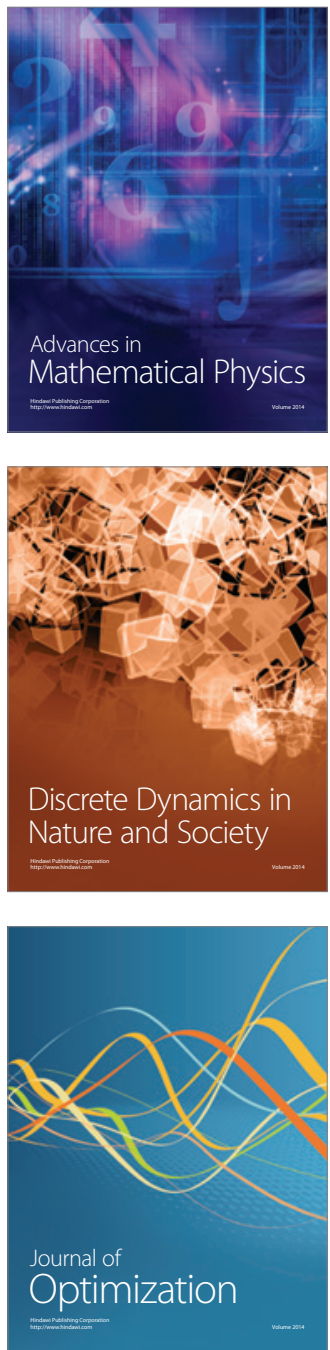